\title{
Highly uniform residual layers for arrays of 3D nanoimprinted cavities in Fabry-Pérot-filter-array-based nanospectrometers
}

\author{
Imran Memon ${ }^{1} \cdot$ Yannan Shen $^{1} \cdot$ Abdullah Khan $^{1} \cdot$ Carsten Woidt $^{1}$ • \\ Hartmut Hillmer ${ }^{1}$
}

Received: 4 May 2015/Accepted: 2 June 2015/Published online: 12 June 2015

(c) The Author(s) 2015. This article is published with open access at Springerlink.com

\begin{abstract}
Miniaturized optical spectrometers can be implemented by an array of Fabry-Pérot (FP) filters. FP filters are composed of two highly reflecting parallel mirrors and a resonance cavity. Each filter transmits a small spectral band (filter line) depending on its individual cavity height. The optical nanospectrometer, a miniaturized FPbased spectrometer, implements 3D NanoImprint technology for the fabrication of multiple FP filter cavities in a single process step. However, it is challenging to avoid the dependency of residual layer (RL) thickness on the shape of the printed patterns in NanoImprint. Since in a nanospectrometer the filter cavities vary in height between neighboring FP filters and, thus, the volume of each cavity varies causing that the RL varies slightly or noticeably between different filters. This is one of the few disadvantages of NanoImprint using soft templates such as substrate conformal imprint lithography which is used in this paper. The advantages of large area soft templates can be revealed substantially if the problem of laterally inhomogeneous RLs can be avoided or reduced considerably. In the case of the nanospectrometer, non-uniform RLs lead to random variations in the designed cavity heights resulting in the shift of desired filter lines. To achieve highly uniform RLs, we report a volume-equalized template design with the lateral distribution of 64 different cavity heights into several units with each unit comprising four cavity heights. The average volume of each unit is kept constant to obtain
\end{abstract}

Imran Memon

memon@ina.uni-kassel.de

1 Institute of Nanostructure Technologies and Analytics, University of Kassel, Heinrich-Plett-Str. 40, 34132 Kassel, Germany uniform filling of imprint material per unit area. The imprint results, based on the volume-equalized template, demonstrate highly uniform RLs of $110 \mathrm{~nm}$ thickness.

Keywords Nanospectrometer - Fabry-Pérot filter . 3D nanoimprint · UV-substrate conformal imprint lithography $\cdot$ Residual layer

\section{Introduction}

Low cost and miniaturized FP-based optical spectrometers are attractive for modern sensing systems e.g., for medical technologies, safety and security, process monitoring, etc. The optical nanospectrometer, a miniaturized spectrometer, is based on static FP filter arrays with 3D nanoimprinted cavities and a corresponding detector array where each filter transmits a distinct spectral filter line depending on its individual cavity thickness (Wang et al. 2013).

A Fabry-Pérot (FP) filter comprises two highly reflecting dielectric mirrors implemented as Distributed Bragg Reflectors (DBRs) and a transparent polymer cavity with a defined thickness which determines the spectral position of the characteristic filter line of each single filter. Each DBR is based on multiple quarter wave thickness layers of alternating materials and produces a highly reflective band around center wavelength $\left(\lambda_{c}\right)$, which is referred to as stopband. Introducing a cavity of halfwave thickness between two DBRs allows a narrow filter line inside the stopband to pass through the filter. The spectral position of the filter line depends on the height of a cavity. Therefore, fabrication of precise cavity heights with sub-nm spatial resolution is required to achieve high spectral resolution, low filter line width, and small spectral filter line separation. 
Fig. 1 a Schematic of a static nanospectrometer with three DBR mirrors of different central wavelengths and 12 different cavities and $\mathbf{b}$ the differing heights of the cavities produce 12 different filter lines

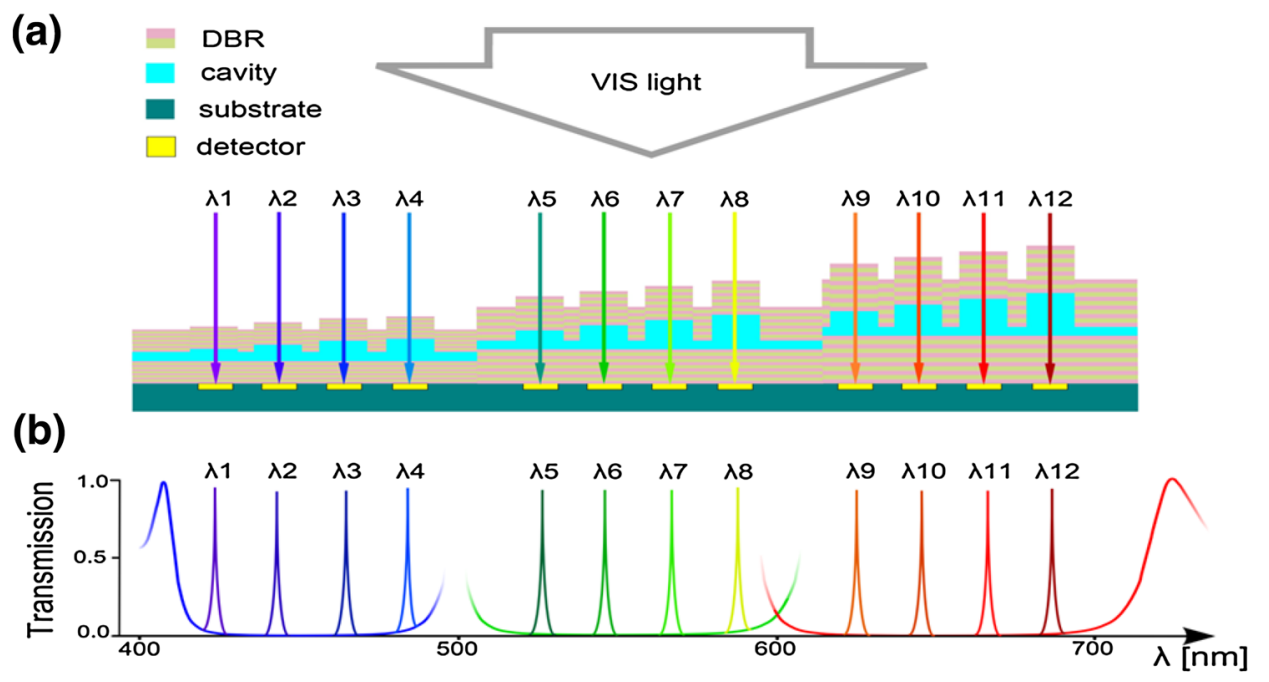

UV-Substrate Conformal Imprint Lithography (UVSCIL), a large area 3D NanoImprint methodology, provides the possibility to structure multiple arrays of FP filter cavities with different heights in a single step (Albrecht et al. 2012; Ji et al. 2010). Figure 1 shows 12 FP filters with distinct cavity heights and their resulting filter lines.

One of the main challenges in implementing the 3D NanoImprint technology is to control RL which is always encountered in processes not using small hard templates. The creation of a RL has an influence on the height of the individual filter cavities thereby resulting in the spectral shift of desired filter lines. The thickness of the RL can be non-uniform, depending on the geometry of the template patterns and the imprint conditions. The inhomogeneous RL thickness can result in a loss of critical dimension control of the patterns during plasma etching process.

The diversity of FP filter cavity heights on a 3D NanoImprint template causes a random and non-uniform variation in the RL below the cavity structures (Bhushan 2010; Bogdanski et al. 2004; Taylor and Boning 2010). The cavity patterns with different heights require different resist volumes for structure filling which lead to the rise of the inhomogeneous RLs using flexible templates as shown in Fig. 2. Therefore, obtaining highly uniform RLs is important for the fabrication of precise cavity heights since the additional uniform RL thickness must be removed by a plasma etching process or compensated in the design of the filter cavities.

To achieve thin and uniform RLs for the nanoimprinted structures with diverse pattern densities, different approaches are known from literature. First, dummy patterns are introduced on a template to homogenize pattern density (Lazzarino et al. 2004). But, the addition of the dummy patterns is not always desirable due to the limited design freedom on the NanoImprint template. The other approach is the use of combined-nanoimprint-and-photolithography

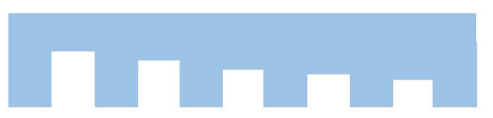

SCIL Template

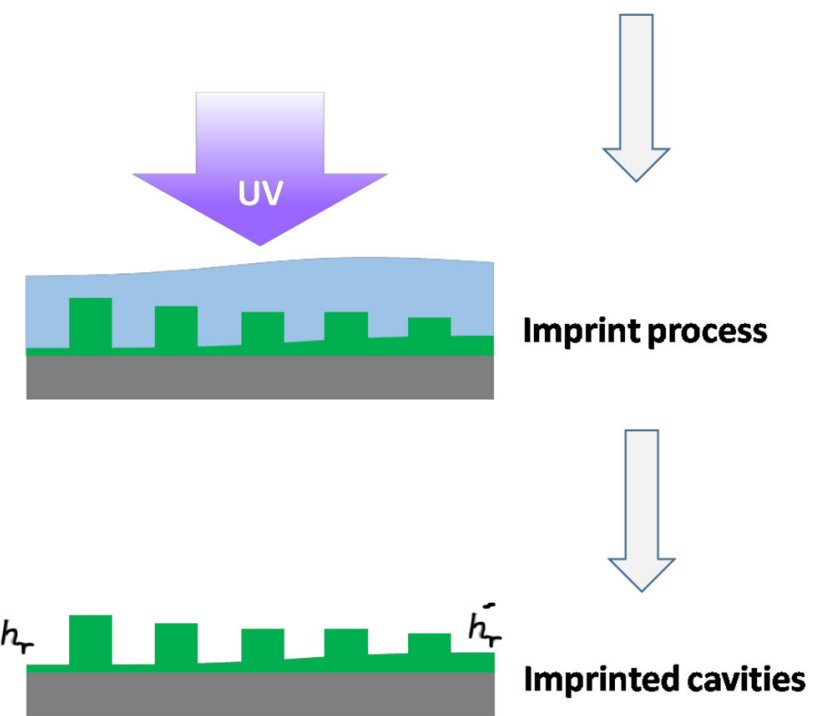

Fig. 2 Non-uniformity of the residual layer due to 3D cavity patterns with different heights on a 3D SCIL template

to imprint patterns without residual layer (Cheng and Guo 2004). Using $\mathrm{SiO}_{2}$ templates partly covered with multiple metal areas, this approach is based on a hybrid-mask-mold which is made from UV transparent material with lightblocking metal layer on top of the protrusions. The hybrid template for the combined-nanoimprint-and-photolithography requires relatively more fabrication steps and, therefore, involving more cost than a single imprint step. The third approach to achieve uniform RLs is the use of an optimized UV-NanoImprint lithography with drop-on-demand method by which droplets of UV-curable resist are supplied according to the local template capacity. The 
controlled dispense of the resist to match up with the local template pattern is employed using step and flash UVNanoImprint lithography (Colburn et al. 1999). The limitation of this solution is the requirement of a dispensing system which is not applicable to all NanoImprint tools. Besides, the dispense process is expensive and time consuming at wafer level. The fourth method is to optimize template design in a manner to compensate the volume differences of different density patterns. This approach offers a cost-effective and promising solution.

In this work, we report a new template design (called volume-equalized template in this work) in which the volume differences of individual FP filter cavities are balanced by arranging them into several units with each unit comprising 4 cavities. The average volume of 4 cavities in each unit is kept constant to achieve uniform filling of the imprint resist per unit area. A 3D template based on the distribution of 64 cavities in several volume-equalized units is fabricated and implemented for UV-SCIL NanoImprint process. Imprint results are characterized below for the quantitative evaluation of RL thickness. An array of FP filters fabricated using the volume-equalized template is characterized to prove uniform RL thickness from a linear relationship between the cavity heights and the corresponding spectral positions of their filter lines.

\section{Experimental work}

\section{Design and fabrication process of 3D templates with volume-equalized distribution}

\section{Template design}

The calculation and distribution of FP filter cavities in volume-equalized units is performed with the help of a mathematical program which is developed in Matlab ${ }^{\circledR}$. The designed SCIL template comprises 64 filter cavities with heights ranging from 30 to $181.2 \mathrm{~nm}$ and a common vertical step difference of $2.4 \mathrm{~nm}$. The cavity structures have identical lateral dimension of $40 \times 40 \mu \mathrm{m}$ and this size of cavity is sufficient for reliable results from FP filters. The desired filter cavities are arranged into 16 basic units with each unit comprising 4 different cavity heights. Each unit has the same average volume which is approx. equal to the mean value of all the 64 different heights (i.e., $105 \mathrm{~nm}$ ) as shown in Fig. 3.

More importantly, the average height of 8 filter cavities in each row is also kept identical. Figure 4 shows a comparison of all possible units before and after the arrangement. The units of filter cavities, which are arranged in an ascending order, show different average volume (the rising blue dotted line). The average height goes on increasing in every following unit. The units, which are distributed and

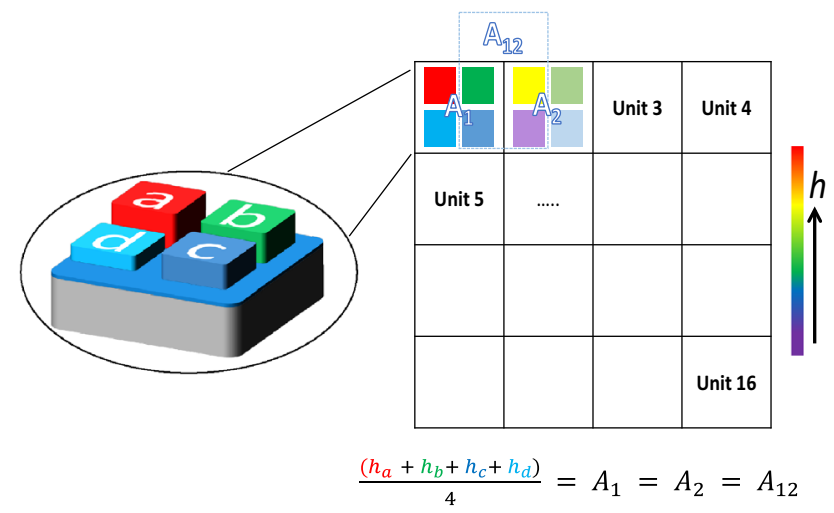

Fig. 3 The distribution of 64 cavities with different heights into units of 4 cavities with each unit having the identical average volume

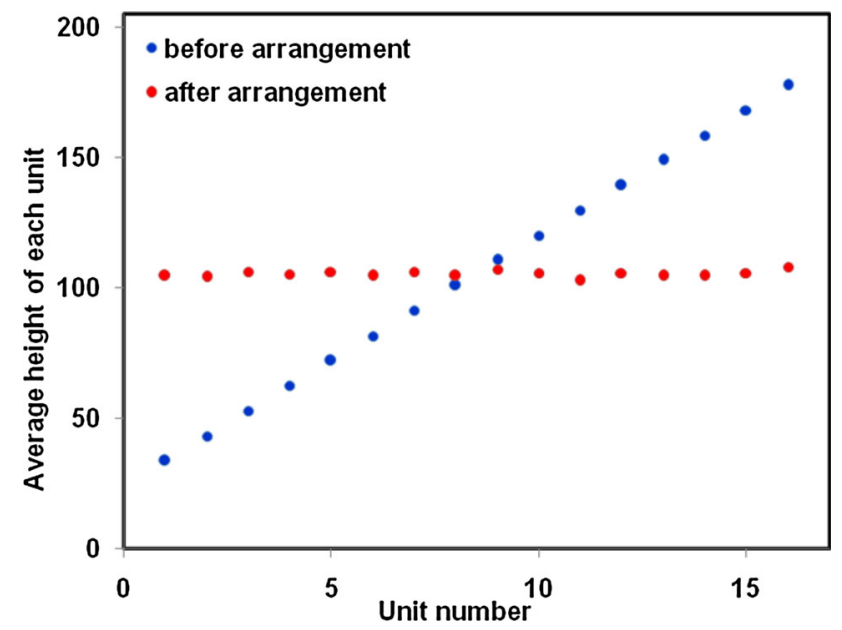

Fig. 4 The arrangement of units in an ascending order and in volume-equalized manner after new arrangement

arranged in the volume-equalized manner, show a uniform average volume (the red dotted line).

The individual units of the cavity heights are not physically isolated but they are arranged into an array of 8 rows and 8 columns. The uniform filling factor of the heights in each unit can be affected by the cavity heights in the surrounding units which may also lead to the rise of non-uniform RLs. Therefore, additional units of 4 cavity heights between the adjacent units are considered in the same manner as the basic units. Table 1 illustrates the distribution of the designed filter cavities in two basic units and also their corresponding approximate average heights. Moreover, the approximated average height of the additional unit between the basic units is also calculated in the table.

\section{Template fabrication process}

Based on the volume-equalized template design, a 3D master template is developed on a 2 -inch gallium arsenide wafer. The master template with 64 different cavity heights 
Table 1 The table shows the distribution of eight different cavity heights in two units and their corresponding approximate average heights

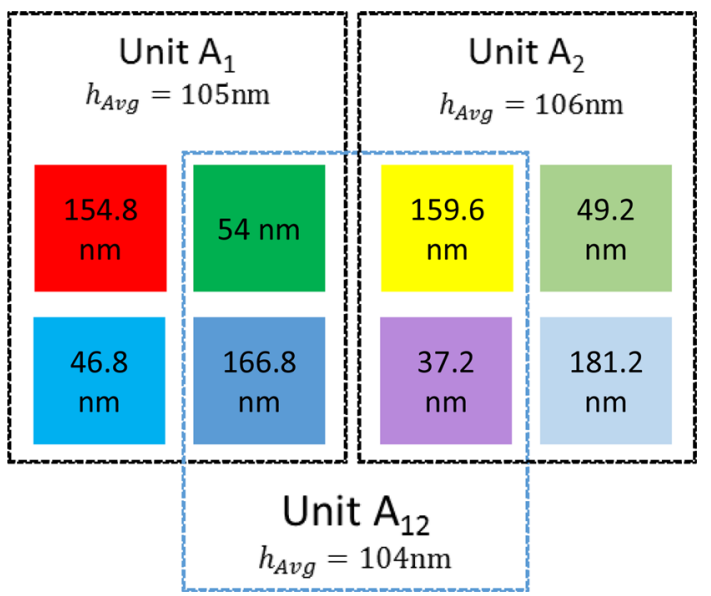

is fabricated using seven steps of photolithography and dry etching in a digital manner. The lithography is performed with mask aligner 4 from SUSS MicroTec and dry etching is carried out using Oxford plasmalab 80+ RIE systems from Oxford Instruments. Later, a 3D SCIL template with negative cavity structures is replicated from the hard master template on the hybrid PDMS stamp using master replication toolkit. The details related to the fabrication processes for the 3D templates are explained somewhere else (Wang et al. 2013).

\section{Imprint process and residual layer measurement}

The UV-SCIL process is performed with mask aligner 6 from SUSS MicroTec using mr-UVCuro06 (Microresist, Germany) as imprint material on a 1 inch glass substrate. The glass substrate is first cleaned with acetone and isopropanol. Then the substrate is placed on a hotplate for dehydration. Afterwards, an adhesion promoter, Ti-Prime from Micro chemicals, is spin coated. The imprint material is spin coated on the substrate at $3000 \mathrm{rpm}$ to obtain a uniform coating of approximately $250 \mathrm{~nm}$ thickness. After the spin coating process, the substrate is again placed on a hotplate for soft-bake at $80{ }^{\circ} \mathrm{C}$ for $60 \mathrm{~s}$. Then UV-SCIL process is performed with MA6 using $180 \mathrm{~s}$ of process delay and $40 \mathrm{~s}$ of exposure time for the filling and curing of the filter cavity structures.

For the quantitative measure of the RL, some part of the imprinted polymer is removed down to the substrate using $\mathrm{O} 2$ plasma etching at $120{ }^{\circ} \mathrm{C}$ for $15 \mathrm{~min}$. Then a thin layer of aluminum $(50 \mathrm{~nm})$ is deposited on the transparent nanoimprinted cavities by physical vapor deposition (PVD). The aluminum layer can be removed by an aluminum etchant after the WLI measurements without affecting the polymer cavity structures. Subsequently, the surface profiles of the nanoimprinted filter cavities and their underlying RL are characterized with white light interferometry (WLI) (Wang et al. 2013).

\section{Fabrication and characterization process for Fabry-Pérot filter arrays}

An array of 64 FP filters, based on two identical DBRs and different cavity heights, is fabricated. First, bottom DBRs with 9.5 periods of $\mathrm{SiO}_{2} / \mathrm{Si}_{3} \mathrm{~N}_{4}$ are deposited for a center wavelength of $500 \mathrm{~nm}$ on a glass substrate by plasma-enhanced chemical vapor deposition (PECVD) process. Then UV-SCIL process is performed using 3D NanoImprint template to imprint 64 different polymer cavity heights on the bottom DBRs. Finally, top DBRs are deposited on the nanoimprinted filter cavities. The optical characterization of the FP filter array for the transmission spectra is carried out with a microscope spectrometer setup which comprises a source of white light, a microscope, a series of optical elements, and optical spectrum analyzer (Mai et al. 2012). The results of the transmission spectra are related to their corresponding cavity heights to determine uniformity in the RL.

\section{Results and discussion}

\section{Evaluation of 3D templates with volume-equalized design}

Using the volume-equalized template design, the desired 3D master template has been successfully prepared using multiple steps of photolithography and reactive ion etching. Figure 5 shows WLI measurement of the topography of 3D filter cavities with different heights on the master template. The variations in the cavity heights can be evaluated on the basis of the differences in their color. The fabricated cavities indicate an average variation of $3 \%$ in the vertical heights from the designed cavities. However, the average height for individual units is close to the mean value of 64 heights (i.e., 105) with a deviation of 2-3 nm.

The replicated SCIL template contains an array of $8 \times 8$ negative cavities corresponding to the positive cavity heights on the master template in the mirror symmetry as shown in Fig. 6. The replication of the cavities from the master template on the SCIL template is not very precise and the replicated cavity depths have an average shrinkage of $4 \%$ as compared to the cavity heights on the master template. Moreover, the average height of 64 cavity depths on the SCIL template is measured to be $100.5 \mathrm{~nm}$.

\section{Imprint results and evaluation of residual layer}

Using the volume-equalized SCIL template, an array of 64 different cavity heights is imprinted on a UV-curable 

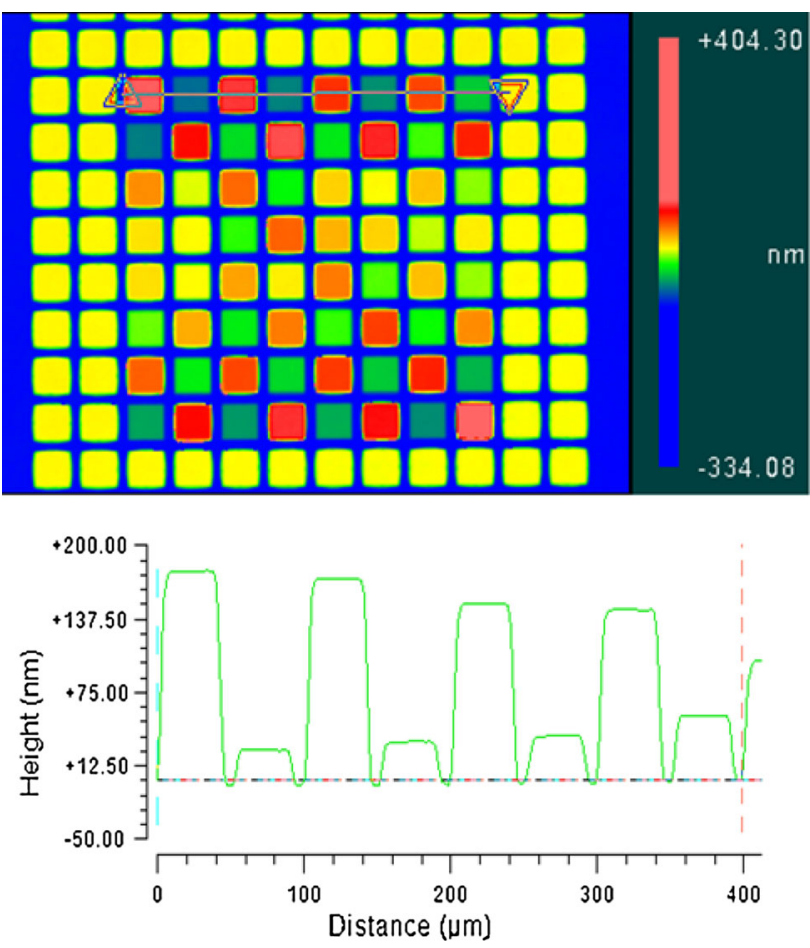

Fig. 5 WLI measurement of topography of 3D cavity structures on GaAs master template (top) and surface profile of first 8 cavities with different heights (bottom)
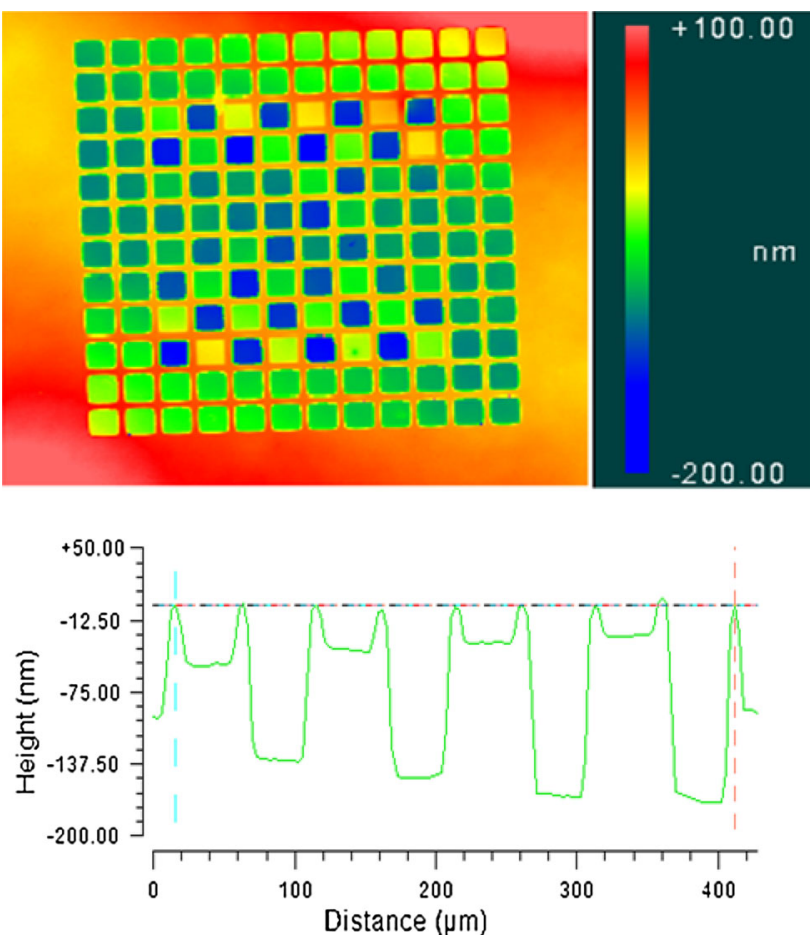

Fig. 6 WLI measurement of topography of 3D cavity structures on SCIL Template (top) and surface profile of first 8 cavities with different depths (bottom)
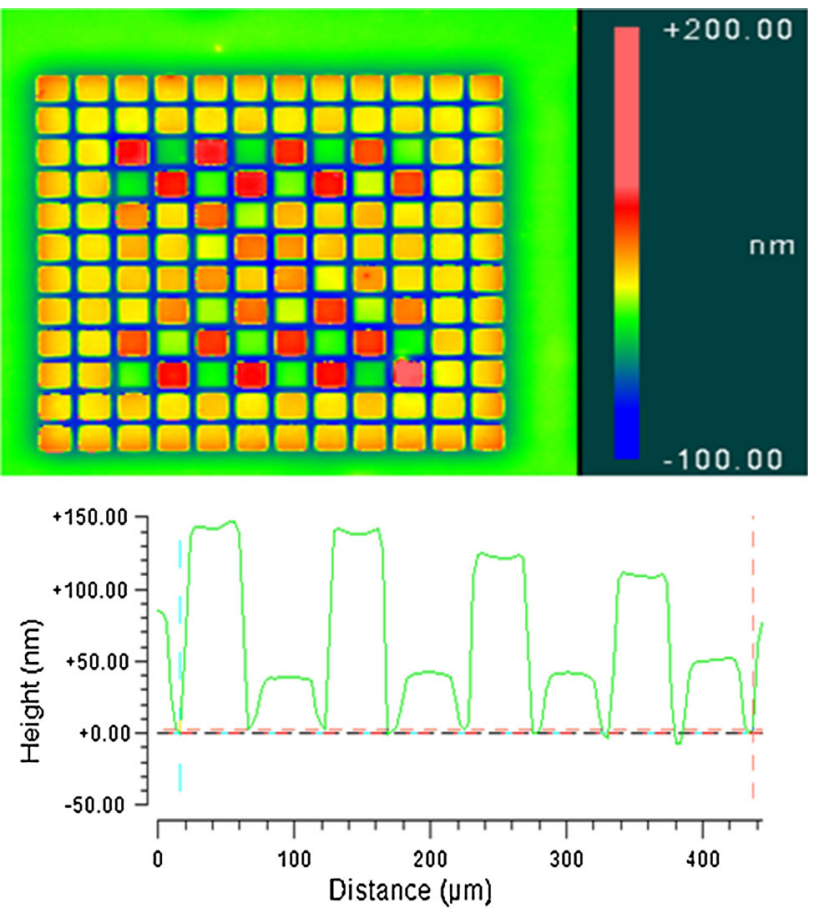

Fig. 7 WLI measurement: Surface topography of 64 cavity structures, imprinted with mr-UVCur06, indicates visible color differences for different cavity heights and similar color for uniform distribution of RL around the cavities (top), surface profile of first 8 cavity heights (bottom)

polymer. Figure 7 shows WLI characterization of the nanoimprinted cavity heights on mr-UVCur06 polymer.

The profile of the RLs for different cavity heights in the array can be evaluated on the basis of visual inspection of the WLI characterization results. The differences in the color for the $3 \mathrm{D}$ cavities indicate the variations in their heights. The checkered area (here blue) between the filters in the array, comprising 64 different heights, shows similar color (blue) in the gaps of the cavity structures. This similarity in color indicates uniformity in the thickness of the RLs across all the different cavity heights.

In order to investigate the imprint uniformity and to quantify thickness of the RLs for different cavity heights, a part of the imprinted array has been stripped off from the polymer down to the substrate with $\mathrm{O}_{2}$ plasma etching. Then the RL is measured for the individual cavities with reference to the substrate. Figure 8 shows a WLI profile of three different cavity heights which indicate nearly the same measure of their RLs (i.e., $110 \mathrm{~nm}$ ) with a deviation of 2-3 nm. This proves that highly uniform RLs are obtained using the volume-equalized template design. The variations in the uniformity of the RLs occur mainly because the cavity heights fabricated on the master template slightly differ from the desired heights. Therefore, the 

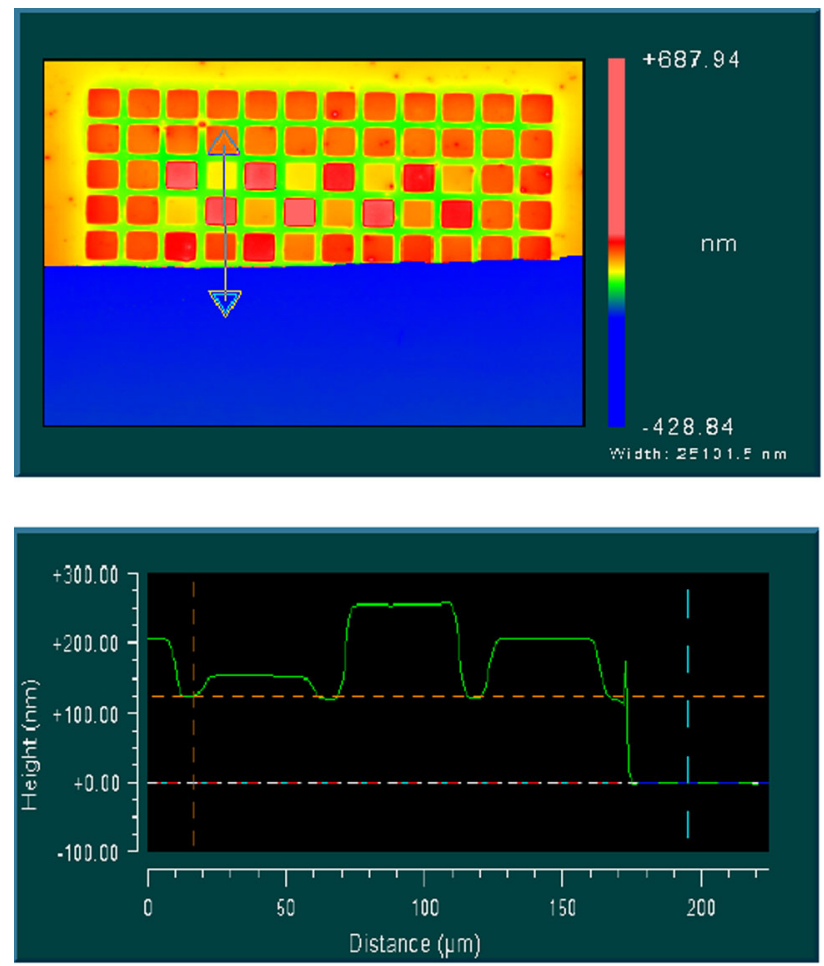

Fig. 8 WLI measurement of residual thickness for different cavities with reference to the substrate (top). The surface profile of three different cavity heights indicates the same measure of RL (bottom)

reported non-uniformity can be removed by the precise fabrication of cavity heights.

\section{Optical characterization of Fabry-Pérot filter arrays}

Using the volume-equalized SCIL template, the polymer cavities are successfully implemented on the bottom DBRs to fabricate an array of 64 different FP filters. The fabricated FP filter array has been characterized to determine the spectral positions of the filter lines for the individual FP filters using the microscope spectrometer setup. Figure 9 shows the optical characterization of 15 different FP filters based on the PECVD DBRs with $550 \mathrm{~nm}$ center wavelength and mr-UVCur06 polymer cavities. The implementation of 64 different cavities with a vertical step difference of $2.4 \mathrm{~nm}$ on the SCIL template is to cover a large range of filter lines within the whole visible range (400-700 nm).

But the spectral width of the stopband from the PECVD DBR, which is limited to approx. $100 \mathrm{~nm}$, cannot accommodate filter lines of all 64 FP filters. It can be seen from the figure that the filter lines from 15 FP filters, which are nearly equidistant, are sufficient for a single PECVD DBR.

The uniformity in the RL thickness can also be evaluated on the basis of optical characterization of FP filters.

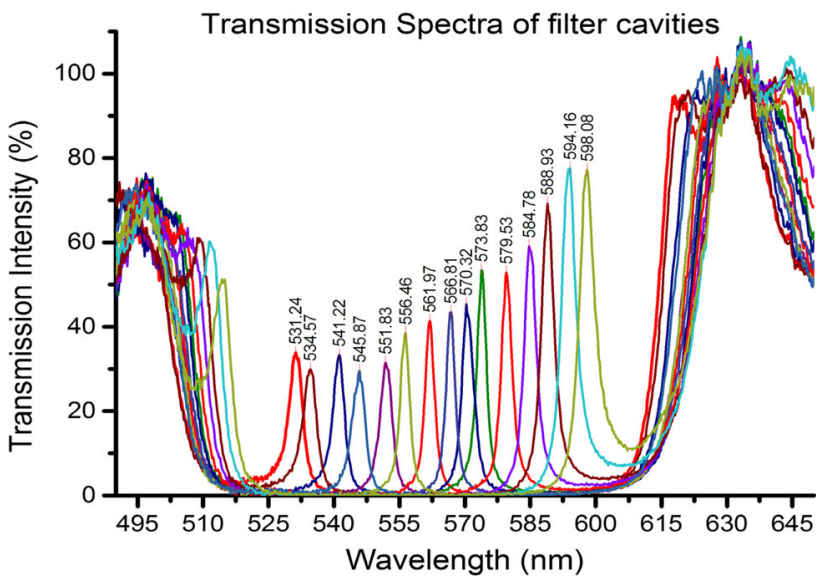

Fig. 9 Transmission spectra of 15 FP filters based on $\mathrm{SiO}_{2} / \mathrm{Si}_{3} \mathrm{~N}_{4}(9.5$ periods) PECVD DBRs and mr-UVCu06 cavities

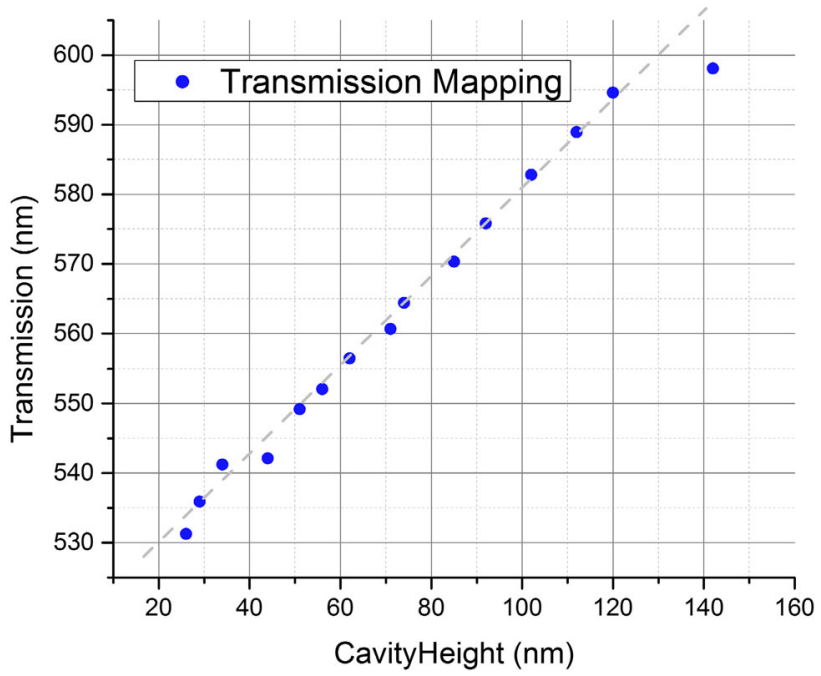

Fig. 10 Mapping of cavity heights and filter lines for 15 FP filters based on $\mathrm{SiO}_{2} / \mathrm{Si}_{3} \mathrm{~N}_{4}$ (9.5 periods) PECVD DBRs and mr-UVCur06 cavities

The mapping of the filter lines to their corresponding cavity heights produces a nearly linear trend as shown in Fig. 10. This indicates that the uniform increment in the cavity heights produces a corresponding uniform shift in the spectral position of the filter lines. Thus, the uniformity in the RL is obtained in the FP filter array based on the new template design. However, some deviations can be seen in the mapping results. This slight variation in the linear mapping is mainly because of two reasons i.e., (1) there is a fabrication error with an average deviation of $3 \%$ in the vertical heights as compared to the desired cavity heights, (2) the filter lines do not depend on the cavity thickness alone but the other factors such as refractive index of the cavity material, etc., also influence the characterization results. Therefore, the effects of top DBR deposition on the 
optical properties of the imprinted polymer cavities need further investigation.

In the following, our results are evaluated in the context of other techniques related to the field of RLs.

The easiest solution to remove the RL in 2D NanoImprint is the overall plasma treatment of the whole developed resist structure. The etching time is chosen to just remove all the RLs. This technique cannot be applied if the structured 3D resist is a functional optical layer as in our nanospectrometer. With plasma etching, it is nearly impossible to control the critical dimensions of the 3D functional features in the presence of inhomogeneous RLs. In case of optical applications, first, the required interface of very high optical quality (abruptness and smoothness) would degrade during the etching. Second, a RL varying in thickness laterally cannot be removed optimally.

Since it is essential that the pattern density on the template should be constant to achieve uniform RLs, therefore, additional patterns are proposed on the NanoImprint template to compensate the density variations of the individual patterns. However, the inclusion of specially designed reservoirs (dummy structures) used to accumulate and store resist volume has many disadvantages. The addition of the non-functional dummy structures on the template might increase the imprint time thereby affecting the throughput of the imprint process. Moreover, the dummy features may be not desirable in all cases due to limited design freedom on the template.

The combined-nanoimprint-photolithography method uses a hybrid mold-mask template to handle the non-uniform RLs (Cheng and Guo 2004). The hybrid template is partly covered with a metal layer on the top of template protrusion patterns in order to prevent the underlying resist from UV exposure and to subsequently remove the unexposed resist in a developer solution. This method has also its limitations. First, it removes the resist layer underneath the metal part of the template following a development process, whereas the RL problem below the cavities is not addressed and solved. Second, the fabrication of the hybrid mold-mask template and the development process after NanoImprint process require several additional processing steps.

The drop-on-demand method offers a comprehensive and reliable solution to cope with the pattern density variations and eliminate the RL in a NanoImprint process (Kim et al. 2006). However, this method is implemented in only specific NanoImprint techniques such as step and flash NanoImprint lithography. These NanoImprint techniques require a dispensing system to adjust the imprint resist according to the local capacity of the template patterns. One of the major limitations of the dispensing method is the relatively low throughput which is a consequence of using multiple stepping and UV flashes to complete the whole imprint process.

Our volume-equalized template design, which is independent of a NanoImprint technique, offers a fast and costeffective solution for obtaining stable cavity patterns with thin and uniform RLs in a single process step. The template fabrication process is very straightforward and is performed without any restriction on adding non-functional structures. Using the volume-equalized soft templates, the NanoImprint process can be implemented over large areas with a single exposure step. The imprint resist spin coated on a substrate can be evenly distributed at each unit area and, thus, the resulting RLs across diverse density patterns can be highly uniform in the 3D NanoImprint process.

\section{Conclusion}

The variations in the RL resulting from diverse FP filter cavity heights during UV-SCIL NanoImprint process are experimentally investigated. To control pattern dependency of the RL for different cavity heights in a FP filter array, a volume-equalized 3D SCIL template is designed and fabricated. WLI characterization of the imprint results from the volume-equalized SCIL template demonstrates a high uniformity in the RL thickness (i.e., $110 \mathrm{~nm}$ ) across all the different 3D cavity patterns. However, a deviation of $2-3 \mathrm{~nm}$ in the RL around the cavity heights is observed. This sub-nm spatial deviation is mainly caused due to $3 \%$ of the variation in the fabricated cavity heights on the master template and general average shrinkage of $4 \%$ in the cavity depths on the replicated 3D SCIL template. The FP filter array fabricated using the SCIL template demonstrates a linear correspondence between the cavity heights and the spectral positions of their filter lines with slight deviations due to the reported reasons. It is also observed that the variation in the cavity heights leads to a shift in the spectral position of the filter lines. For future work, the effects of polymerization shrinkage and top DBRs deposition on the RL will be investigated for stable polymer cavities.

Acknowledgments The authors would like to thank A. Albrecht, A. Istock, and R.Kolli for stimulating scientific discussions and A. Dück for technological support. The financial support of EU 7FP-KBBE project Bread-Guard (Grant No. 613647) is gratefully acknowledged.

Open Access This article is distributed under the terms of the Creative Commons Attribution 4.0 International License (http://creativecommons.org/licenses/by/4.0/), which permits unrestricted use, distribution, and reproduction in any medium, provided you give appropriate credit to the original author(s) and the source, provide a link to the Creative Commons license, and indicate if changes were made. 


\section{References}

Albrecht A, Wang X, Mai HH, Schotzko T, Memon I, Bartels M, Hornung M, Hillmer H (2012) High vertical resolution 3D nanolmprint technology and its application in optical nanosensors. Nonlinear Optics Quantum Optics 43:339-353

Bhushan B (2010) Springer handbook of Nanotechnology, 3rd edn. Springer, Berlin Heidelberg

Bogdanski N, Schulz H, Wissen M, Scheer H-C, Zajadacz J, Zimmer K (2004) 3D-Hot embossing of undercut structures: an approach to micro-zippers. Microelectron Eng 73-74:190-195

Cheng X, Guo LJ (2004) A combined-nanoimprint-and-photolithography patterning technique. Microelectron Eng 71:277-282

Colburn M, Johnson SC, Stewart MD, Damle S, Bailey TC, Choi B, Wedlake M, Michaelson TB, Sreenivasan SV, Ekerdt JG, Willson CG (1999) Step and flash imprint lithography: a new approach to high-resolution patterning. In: Proceedings SPIE, Emerging Lithographic Technologies III, vol 3676

Ji R, Hornung M, Verschuuren MA, van de Laar R, van Eekelen J, Plachetka U, Moeller M, Moormann C (2010) UV enhanced substrate conformal imprint lithography (UV-SCIL) technique for photonic crystals patterning in LED manufacturing. Microelectron Eng 87:963-967

Kim K-D, Jeong J-H, Sim Y-S, Lee E-S (2006) Minimization of residual layer thickness by using the optimized dispensing method in S-FILTM process. Microelectron Eng 83(4-9):847-850

Lazzarino F, Gourgon C, Schiavone P, Perret C (2004) Mold deformation in nanoimprint lithography. J Vac Sci Technol, B 22:3318-3322

Mai HH, Albrecht A, Woidt C, Wang X, Daneker V, Setyawati O, Woit T, Schultz K, Bartels M, Hillmer H (2012) 3D nanoimprinted Fabry-Pérot filter arrays and methodologies for optical characterization. Appl Phys B 107(3):755-764

Taylor H, Boning D (2010) Towards nanoimprint lithography-aware layout design. In: Proceedings SPIE, Design for Manufacturability through Design-Process Integration IV, vol 7641

Wang X, Albrecht A, Mai HH, Woidt C, Meinl T, Hornung M, Bartels M, Hillmer H (2013) High resolution 3D NanoImprint technology: template fabrication, application in Fabry-Pérotfilter-array-based optical nanospectrometers. Microelectron Eng 110:44-51 